MATHEMATICS OF COMPUTATION

Volume 69, Number 230, Pages 839-849

S 0025-5718(99)01100-X

Article electronically published on May 17, 1999

\title{
ON THE SOLUTIONS OF A FAMILY OF QUARTIC THUE EQUATIONS
}

\author{
ALAIN TOGBÉ
}

\begin{abstract}
In this paper, we solve a certain family of diophantine equations associated with a family of cyclic quartic number fields. In fact, we prove that for $n \leq 5 \times 10^{6}$ and $n \geq N=1.191 \times 10^{19}$, with $n, n+2, n^{2}+4$ square-free, the Thue equation

$$
\Phi_{n}(x, y)=x^{4}-n^{2} x^{3} y-\left(n^{3}+2 n^{2}+4 n+2\right) x^{2} y^{2}-n^{2} x y^{3}+y^{4}=1
$$

has no integral solution except the trivial ones: $(1,0),(-1,0),(0,1),(0,-1)$.
\end{abstract}

\section{INTRODUCTION}

We consider the following Thue equation:

$$
\Phi_{n}(x, y)=x^{4}-n^{2} x^{3} y-\left(n^{3}+2 n^{2}+4 n+2\right) x^{2} y^{2}-n^{2} x y^{3}+y^{4}=1,
$$

and we plan to prove that for $n \leq 5 \times 10^{6}$ and $n \geq N=1.191 \times 10^{19}$, with $n$, $n+2, n^{2}+4$ square-free, this equation has only the solutions $(1,0),(-1,0),(0,1)$, $(0,-1)$.

In 1991, L. C. Washington 13 and O. Lecacheux 44 independently studied the units of $\mathbb{Q}(\omega)$, where $\omega$ is a root the polynomial $\Phi_{n}(x, 1)$. Their results were helpful to solve the equation (1.1). For the proof, we use estimates from linear forms in the logarithms of algebraic numbers, techniques from diophantine approximation and new methods of computation.

This paper is based upon the results of our Ph.D. dissertation [12] and is divided into 5 sections. In the second section, we shall give some elementary properties of the above polynomial and recall the result of L. C. Washington and O. Lecacheux about the fundamental system of units of the number field associated with this Thue equation. We shall study the approximative properties of the solutions in the third section. In the last two sections, we solve the equation using respectively the method proposed by M. Mignotte in [6] and the Bilu-Hanrot method (see [2]).

\section{Elementary PRoperties of the Polynomial}

We have the following properties.

(a) $\Phi_{n}( \pm 1,0)=\Phi_{n}(0, \pm 1)=1$. So $(1,0),(-1,0),(0,1),(0,-1)$ are solutions of (1.1) and are called the trivial solutions.

Received by the editor March 3, 1998 and, in revised form, April 28, 1998

1991 Mathematics Subject Classification. Primary 11D25, 11D72, 11D85, 11J86, 11R16, $11 \mathrm{Y} 50$.

Key words and phrases. Quartic equations, equations in many variables, representation problems, linear forms in logarithms, Baker's method, quartic extensions, computer solution of Diophantine equations. 
(b) $\Phi_{n}(x, y)=\Phi_{n}(-x,-y)=\Phi_{n}(y, x)=\Phi_{n}(-y,-x)$, so if $(x, y)$ is a solution of $(1.1)$, so are $(-x,-y),(y, x),(-y,-x)$. Without loss of generality, we shall consider only the solutions $(x, y)$ with $y$ positive.

Lemma 2.1. If $n \neq 0$, then $\Phi(x)=\Phi_{n}(x, 1)$ is irreducible.

Proof. Let $\alpha$ be a root of $\Phi(x)$ and $\eta=\alpha+\frac{1}{\alpha}$. Then, $\eta$ is a root of

$$
z^{2}-n^{2} z-\left(n^{3}+2 n^{2}+4 n+4\right) .
$$

The discriminant of $z^{2}-n^{2} z-\left(n^{3}+2 n^{2}+4 n+4\right)$ is $\Delta=(n+2)^{2}\left(n^{2}+4\right)$, which is not a square for $n \neq 0$. So the quadratic subfield of $\mathbb{K}$ is $k=\mathbb{Q}\left(\sqrt{n^{2}+4}\right)$. Suppose now that $\alpha \in \mathbb{Q}(\eta)$. We know that $\alpha$ is a root of $X^{2}-X \eta+1=0$ for which the discriminant is

$$
\omega:=\eta^{2}-4=\left(n^{3}+2 n^{2}+4 n\right)+n^{2} \eta .
$$

We have $N_{\mathbb{Q}(\eta) / \mathbb{Q}}(\omega)=n^{2}(n+2)^{2}\left(n^{2}+4\right)$. This norm is a square if and only if $n=0$. So $X^{2}-X \eta+1$ is irreducible over $\mathbb{Q}(\eta)$, i.e., $\alpha \notin \mathbb{Q}(\eta)$ if $n \neq 0$.

We know from 4 and 13 that the Galois group of $\mathbb{K}=\mathbb{Q}(\alpha), \alpha$ being a root of $\Phi(x)=\Phi_{n}(x, 1)$, is a cyclic group of order 4 , generated by $\sigma$. Moreover

$$
\Phi(x)=(x-\alpha)(x+\beta)(x-1 / \alpha)(x+1 / \beta),
$$

where $\alpha=\alpha_{1}$ is the largest root of $\Phi(x)$, the other roots being $\alpha_{2}=\sigma\left(\alpha_{1}\right)$ with

$$
\begin{gathered}
\alpha_{2}=\left(n+\frac{1}{n+2}\right)-\left(n^{3}+n^{2}+3 n+\frac{3}{n+2}\right) \alpha_{1}+\left(-n^{2}+n-2+\frac{3}{n+2}\right) \alpha_{1}^{2}+\left(1-\frac{1}{n+2}\right) \alpha_{1}^{3}, \\
\alpha_{3}=\sigma^{2}(\alpha)=1 / \alpha_{1}, \quad \alpha_{4}=\sigma^{3}(\alpha)=1 / \alpha_{2} .
\end{gathered}
$$

For $n \geq 4$, we have the following inequalities for $\alpha_{i}$ :

$$
\begin{aligned}
n^{2}+n+1+\frac{2}{n}-\frac{3}{n^{2}} & <\alpha_{1}<n^{2}+n+1+\frac{2}{n}-\frac{2}{n^{2}}, \\
-n-1-\frac{1}{n}+\frac{1}{n^{3}} & <\alpha_{2}<-n-1-\frac{1}{n}+\frac{2}{n^{2}}, \\
\frac{1}{n^{2}}-\frac{2}{n^{3}} & <\alpha_{3}<\frac{1}{n^{2}}-\frac{1}{n^{3}}, \\
-\frac{1}{n}+\frac{1}{n^{2}}-\frac{1}{n^{3}} & <\alpha_{4}<-\frac{1}{n}+\frac{1}{n^{2}} .
\end{aligned}
$$

Now let $n \neq-1$ be an odd integer such that $n, n+2$ and $n^{2}+4$ are square-free. Then the discriminant of $\mathbb{K}$ is $D=n^{2}(n+2)^{2}\left(n^{2}+4\right)^{3}$ and the fundamental unit of $k$ is

$$
\varepsilon=\frac{n+\sqrt{n^{2}+4}}{2}
$$

We know from [13] that $\left\{1, \varepsilon, \alpha_{1}, \alpha_{2}\right\}$ is a basis for the ring of integers $\mathcal{O}_{\mathbb{K}}$ of $\mathbb{K}$; moreover, $\left\{1, \varepsilon, \alpha_{1}, \varepsilon \alpha_{1}\right\}$ is an integral basis for $\mathbb{K}$, which shows that $\left\{1, \alpha_{1}\right\}$ is a basis for $\mathbb{K}$ over $k$. We have the following result (see [4] or [13]).

Theorem 2.2 (L. C. Washington, O. Lecacheux). When $n, n+2$ and $n^{2}+4$ are square-free, $\left\{\varepsilon, \alpha_{1}, \alpha_{2}\right\}$ is a fundamental system of units of $\mathbb{K}$ when $|n| \geq 3$. When $n=1$, the subgroup $\left\langle-1, \varepsilon, \alpha_{1}, \alpha_{2}\right\rangle$ is of index 2 in the full group of units. 
We shall begin this section with the following result.

Proposition 3.1. Let $(x, y)$ be an integral solution of (1.1); then we have $|y| \geq 2$ or $(x, y)=(1,0),(-1,0),(0,1),(0,-1)$.

Proof. We know that if $y=0$, then $x= \pm 1$. Now suppose $y=1$; then

$$
x=0 \text { or } P(x)=x^{3}-n^{2} x^{2}-\left(n^{3}+2 n^{2}+4 n+2\right) x-n^{2}=0 .
$$

So for $n \geq 1, P(x)$ has three real roots. Moreover, we have

$$
\begin{gathered}
P(-n-1)=n^{2}+3 n+1>0, \quad P(-n-2)=-n^{3}-3 n^{2}-2 n-4<0, \\
P(-1)=n^{3}+4 n+1>0, \quad P(0)=-n^{2}<0, \\
P\left(n^{2}+n+1\right)=-2 n^{3}-4 n^{2}-3 n-1<0, \quad P\left(n^{2}+n+2\right)=n^{4}+n^{3}+3 n^{2}+2 n+4>0 .
\end{gathered}
$$

Then these roots are in $]-n-2,-n-1[, \quad]-1,0[, \quad] n^{2}+n+1, n^{2}+n+2[$, respectively.

Now let $(x, y)$ be a non-trivial solution of (1.1). Then

$$
\Phi_{n}(x, y)=(x-\alpha y)(x+\beta y)\left(x-\frac{1}{\alpha} y\right)\left(x+\frac{1}{\beta} y\right)=1
$$

is the norm of the algebraic unit $\gamma_{1}:=x-\alpha_{1} y \in \mathcal{O}_{\mathbb{K}}^{\times}$, so there are unique integers $u_{0} \in\{0,1\}, u_{1}, u_{2}, u_{3}$ such that

$$
\gamma_{1}=(-1)^{u_{0}} \varepsilon^{u_{1}} \alpha^{u_{2}} \beta^{u_{3}}
$$

We call $u_{0}, u_{1}, u_{2}, u_{3}$ the associated exponents of the solution $(x, y)$. Moreover, we have

$$
\left\{\begin{array}{l}
N_{k / \mathbb{Q}}(\varepsilon)=\varepsilon \sigma(\varepsilon)=-1 \\
\gamma_{i+1}=\sigma\left(\gamma_{i}\right)=x-\alpha_{i+1} y, \quad 1 \leq i \leq 3
\end{array}\right.
$$

So we have

$$
\left\{\begin{aligned}
\left|\gamma_{1}\right| & =\varepsilon^{u_{1}} \alpha^{u_{2}} \beta^{u_{3}} \\
\left|\gamma_{2}\right| & =\varepsilon^{-u_{1}} \beta^{u_{2}} \alpha^{-u_{3}} \\
\left|\gamma_{3}\right| & =\varepsilon^{u_{1}} \alpha^{-u_{2}} \beta^{-u_{3}} \\
\left|\gamma_{4}\right| & =\varepsilon^{-u_{1}} \beta^{-u_{2}} \alpha^{u_{3}}
\end{aligned}\right.
$$

The inequalities for the $\alpha_{i}$ give

$$
-\beta<-\frac{1}{\beta}<\frac{1}{\alpha}<\alpha,
$$

$$
\frac{x}{y}-\alpha<\frac{x}{y}-\frac{1}{\alpha}<\frac{x}{y}+\frac{1}{\beta}<\frac{x}{y}+\beta .
$$

There are four possibilities to obtain the minimum of $\left|\gamma_{i}\right|, 1 \leq i \leq 4$. We shall say that $(x, y)$ is a of type $1,2,3$ or 4 :

- type 1, if all $\gamma_{i}$ 's are positive and $x-\alpha y=\min _{1 \leq i \leq 4}\left\{x-\alpha_{i} y\right\}$;

- type 2 , if all $\gamma_{i}$ 's are negative and $-x-y \beta=\min _{1 \leq i \leq 4}\left\{\left|x-\alpha_{i} y\right|\right\}$;

- type 3 , if $\gamma_{1}, \gamma_{3}$ are negative, $\gamma_{2}, \gamma_{4}$ are positive and $y \frac{1}{\alpha}-x=\min _{1 \leq i \leq 4}\left\{\left|x-\alpha_{i} y\right|\right\}$;

- type 4 , if $\gamma_{1}, \gamma_{3}$ are negative, $\gamma_{2}, \gamma_{4}$ are positive and $y \frac{1}{\beta}+x=\min _{1 \leq i \leq 4}\left\{\left|x-\alpha_{i} y\right|\right\}$.

The above types exhaust all the possibilities. 


\section{PART I}

4.1. Solutions of type 1. Suppose that $(x, y) \in \mathbb{Z}^{2}$ is a non-trivial solution of type 1 .

Lemma 4.1. We have

$$
\begin{aligned}
0 & <\gamma_{1}<y \frac{1}{2 n^{6}} \\
y(\alpha+\beta) & <\gamma_{2}<y\left(\alpha+\beta+\frac{1}{2 n^{6}}\right), \\
y\left(\alpha-\frac{1}{\alpha}\right) & <\gamma_{3}<y\left(\alpha-\frac{1}{\alpha}+\frac{1}{2 n^{6}}\right), \\
y\left(\alpha+\frac{1}{\beta}\right) & <\gamma_{4}<y\left(\alpha+\frac{1}{\beta}+\frac{1}{2 n^{6}}\right) .
\end{aligned}
$$

Proof. We have $y\left|\alpha-\alpha_{i}\right| \leq|x-\alpha y|+\left|x-\alpha_{i} y\right| \leq 2\left(x-\alpha_{i} y\right)$ for $2 \leq i \leq 4$, and then

$$
\frac{1}{x-\alpha_{i} y} \leq \frac{2}{y\left(\alpha-\alpha_{i}\right)}, \quad 2 \leq i \leq 4
$$

So we obtain

$$
x-\alpha y=\prod_{i=2}^{4} \frac{1}{x-\alpha_{i} y} \leq \prod_{i=2}^{4} \frac{2}{\left(\alpha-\alpha_{i}\right) y}=\frac{8}{\Phi^{\prime}(\alpha)} y^{-3},
$$

where $\Phi^{\prime}(\alpha)=\left(\alpha-\alpha_{2}\right)\left(\alpha-\alpha_{3}\right)\left(\alpha-\alpha_{4}\right)$. But $\Phi^{\prime}(\alpha)>n^{6}$, for $n \geq 1$; therefore

$$
\gamma_{1}=x-\alpha y \leq \frac{8}{\Phi^{\prime}(\alpha)} y^{-3}<\frac{8}{y^{3} n^{6}} \leq \frac{1}{n^{6}},
$$

whereupon $0<\frac{x}{y}-\alpha<\frac{1}{y n^{6}} \leq \frac{1}{2 n^{6}}(y \geq 2)$, from which we conclude that

$$
y\left(\alpha-\alpha_{i}\right)<\gamma_{i}<y\left(\alpha-\alpha_{i}+\frac{1}{2 n^{6}}\right), \text { for } 1 \leq i \leq 4 .
$$

So we obtain the desired result.

Let us consider the Siegel identity

$$
0<\frac{\gamma_{4}\left(\alpha_{1}-\alpha_{2}\right)}{\gamma_{2}\left(\alpha_{1}-\alpha_{4}\right)}=1-\frac{\gamma_{1}\left(\alpha_{2}-\alpha_{4}\right)}{\gamma_{2}\left(\alpha_{1}-\alpha_{4}\right)}
$$

so

$$
\alpha^{2 u_{3}} \beta^{-2 u_{2}}\left(\frac{\alpha+\beta}{\alpha+\frac{1}{\beta}}\right)=1+\frac{\gamma_{1}}{\gamma_{2}}\left(\frac{\beta^{2}-1}{\alpha \beta+1}\right) .
$$

Put $A_{1}=2 u_{3}, B_{1}=-2 u_{2}, \lambda_{1}=\frac{\alpha+\beta}{\alpha+\frac{1}{\beta}}, \tau_{1}=\frac{\gamma_{1}}{\gamma_{2}}\left(\frac{\beta^{2}-1}{\alpha \beta+1}\right)$, to obtain the following linear form in logarithms:

$$
\Lambda_{1}=A_{1} \log (\alpha)+B_{1} \log (\beta)+\log \left(\lambda_{1}\right)=\log \left(1+\tau_{1}\right) .
$$

Several results can be deduced from this.

Lemma 4.2. $\Lambda_{1} \neq 0$.

Proof. Because $\tau_{1}>0$.

Lemma 4.3. We have

$$
u_{2}<u_{3}<0 \text { and } 0<\left|A_{1}\right|<B_{1} .
$$


Proof. From the formula (3.2), we obtain

$$
\begin{gathered}
u_{1}=-\frac{c_{2}+c_{4}}{2 \log (\varepsilon)}=\frac{c_{1}+c_{3}}{2 \log (\varepsilon)}, \quad u_{2}=\frac{\left(c_{1}-c_{3}\right) \log (\alpha)+\left(c_{2}-c_{4}\right) \log (\beta)}{2\left[\log ^{2}(\alpha)+\log ^{2}(\beta)\right]}, \\
u_{3}=\frac{\left(c_{1}-c_{3}\right) \log (\beta)-\left(c_{2}-c_{4}\right) \log (\alpha)}{2\left[\log ^{2}(\alpha)+\log ^{2}(\beta)\right]},
\end{gathered}
$$

with $c_{i}=\log \left|\gamma_{i}\right|$. So $c_{1}<c_{3}<c_{4}<c_{2}, c_{1}-c_{3}<0$ and $c_{2}-c_{4}>0$, for $n \geq 3$; then $u_{3}<0$ and $A_{1}<0$. Moreover, we have

$$
\begin{aligned}
& \left(c_{1}-c_{3}\right) \log (\alpha)+\left(c_{2}-c_{4}\right) \log (\beta) \\
& \quad<\log (\beta) \log \left(\frac{\alpha+\beta+\frac{1}{2 n^{6}}}{\alpha+\frac{1}{\beta}}\right)-\log (\alpha) \log \left(2 n^{6}\left(\alpha-\frac{1}{\alpha}\right)\right) .
\end{aligned}
$$

The right hand side of the inequality is negative for $n \geq 3$. Therefore $u_{2}<0$ and $B_{1}>0$, for $n \geq 3$.

Finally, the expression $\log \left(\frac{\gamma_{1}}{\gamma_{3}}\right)(\log (\alpha)-\log (\beta))+\log \left(\frac{\gamma_{2}}{\gamma_{4}}\right)(\log (\alpha)+\log (\beta))$ is less than

$$
\begin{array}{r}
-\log \left(2 n^{6}\left(\alpha-\frac{1}{\alpha}+\frac{1}{2 n^{6}}\right)\right)(\log (\alpha)-\log (\beta)) \\
+\log \left(\frac{\alpha+\beta+\frac{1}{2 n^{6}}}{\alpha+\frac{1}{\beta}}\right)(\log (\alpha)+\log (\beta)),
\end{array}
$$

which is negative for $n \geq 3$; so $u_{2}-u_{3}<0$, i.e., $u_{2}<u_{3}<0,0<-2 u_{3}<-2 u_{2}$, i.e., $0<\left|A_{1}\right|<B_{1}$.

We use the Baker-Wüstholz theorem, (see 1], page 20) to obtain a lower bound of $\Lambda_{1}$.

Lemma 4.4. We have

$$
\log \left(\Lambda_{1}\right)>-2^{33} \times 3^{7} \times \log (24) \log ^{3}(\alpha \beta) \log \left(B_{1}\right)
$$

Proof. We have

$$
\begin{gathered}
h^{\prime}(\alpha)=h^{\prime}(\beta)=\frac{1}{4} \log (\alpha \beta), \\
h^{\prime}\left(\lambda_{1}\right)=\frac{1}{4} \log \left(\frac{\alpha+\beta}{\alpha+1 / \beta} \cdot \frac{\alpha+1 / \beta}{1 / \beta+1 / \alpha}\right)=\frac{1}{4} \log (\alpha \beta),
\end{gathered}
$$

because the conjugates of $\lambda_{1}$ are

$$
\begin{aligned}
\lambda_{1}^{(1)}=\frac{\alpha+\beta}{\alpha+1 / \beta}>1, & \lambda_{1}^{(2)}=\frac{\beta+1 / \alpha}{\beta+\alpha}<1, \\
\lambda_{1}^{(3)}=\frac{1 / \alpha+1 / \beta}{1 / \alpha+\beta}<1, & \lambda_{1}^{(4)}=\frac{1 / \beta+\alpha}{1 / \beta+1 / \alpha}>1 ;
\end{aligned}
$$

and because $B=\max \left\{-2 u_{2},-2 u_{3}, 1\right\}=-2 u_{2}=B_{1}$ and $C(3,4)=3^{7} \times 2^{39} \log (24)$. So we obtain the desired result.

The following result gives us an upper bound for $\log \left(\Lambda_{1}\right)$.

Lemma 4.5. For $n \geq 4$, we have

$$
\log \left(\Lambda_{1}\right)<-1.4 B_{1} \log (\alpha) .
$$


Proof. We know that $\Lambda_{1}=\log \left(1+\tau_{1}\right) \leq \tau_{1}=\frac{\gamma_{1}}{\gamma_{2}}\left(\frac{\beta^{2}-1}{\alpha \beta+1}\right)$, so

$$
\log \left(\Lambda_{1}\right) \leq \log \left(\frac{\gamma_{1}}{\gamma_{2}}\left(\frac{\beta^{2}-1}{\alpha \beta+1}\right)\right)=-\log \left(\frac{\gamma_{2}}{\gamma_{1}}\left(\frac{\alpha \beta+1}{\beta^{2}-1}\right)\right) .
$$

We have to show that $1.4 B_{1} \log (\alpha)<\log \left(\frac{\gamma_{2}}{\gamma_{1}}\right)+\log \left(\frac{\alpha \beta+1}{\beta^{2}-1}\right)$. According to the expression of $B_{1}$, it remains to show that

$$
\begin{aligned}
& \frac{1.4 \log ^{2}(\alpha)}{\log ^{2}(\alpha)+\log ^{2}(\beta)} \log \left(\frac{\gamma_{3}}{\gamma_{1}}\right)+\frac{1.4 \log (\alpha) \log (\beta)}{\log ^{2}(\alpha) !+\log ^{2}(\beta)} \log \left(\frac{\gamma_{4}}{\gamma_{2}}\right) \\
& \quad<\log \left(\frac{\gamma_{2}}{\gamma_{1}}\right)+\log \left(\frac{\alpha \beta+1}{\beta^{2}-1}\right),
\end{aligned}
$$

i.e.,

$$
\begin{aligned}
& \frac{1.4 \log (\alpha) \log (\beta)}{\log ^{2}(\alpha)+\log ^{2}(\beta)} \log \left(\frac{\gamma_{4}}{\gamma_{2}}\right) \\
& \quad<\log \left(\frac{\alpha \beta+1}{\beta^{2}-1}\right)+\log \left(\frac{\gamma_{2}}{\gamma_{3}}\right)+\left(1-\frac{1.4 \log ^{2}(\alpha)}{\log ^{2}(\alpha)+\log ^{2}(\beta)}\right) \log \left(\frac{\gamma_{3}}{\gamma_{1}}\right) .
\end{aligned}
$$

We know that

$$
\log \left(\frac{\gamma_{4}}{\gamma_{2}}\right)<\log \left(\frac{\alpha+\frac{1}{\beta}+\frac{1}{2 n^{6}}}{\alpha+\beta}\right), \quad \log \left(\frac{\alpha+\beta}{\alpha-\frac{1}{\alpha}+\frac{1}{2 n^{6}}}\right)<\log \left(\frac{\gamma_{2}}{\gamma_{3}}\right),
$$

and

$$
\log \left(2 n^{6}\left(\alpha-\frac{1}{\alpha}\right)\right)<\log \left(\frac{\gamma_{3}}{\gamma_{1}}\right) .
$$

Then we must prove that

$$
\frac{1.4 \log (\alpha) \log (\beta)}{\log ^{2}(\alpha)+\log ^{2}(\beta)} \log \left(\frac{\alpha+\frac{1}{\beta}+\frac{1}{2 n^{6}}}{\alpha+\beta}\right)
$$

is less than

$$
\log \left(\frac{\alpha \beta+1}{\beta^{2}-1}\right)+\log \left(\frac{\alpha+\beta}{\alpha-\frac{1}{\alpha}+\frac{1}{2 n^{6}}}\right)+\left(1-\frac{1.4 \log ^{2}(\alpha)}{\log ^{2}(\alpha)+\log ^{2}(\beta)}\right) \log \left(2 n^{6}\left(\alpha-\frac{1}{\alpha}\right)\right),
$$

but one can check that this is indeed the case.

Using the last two results, we obtain

$$
1.4 B_{1} \log (\alpha)-2^{33} \times 3^{7} \times \log (24) \log ^{3}(\alpha \beta) \log \left(B_{1}\right)<0 .
$$

Put

$$
K_{1}=\frac{2^{33} \times 3^{7} \times \log (24) \log ^{3}(\alpha \beta)}{1.4 \log (\alpha)}, \quad G_{1, n}(x)=x-K_{1} \log (x) ;
$$

then we have

$$
\begin{gathered}
G_{1, n}\left(B_{1}\right)<0, \quad G_{1, n}^{\prime}(x)=1-\frac{K_{1}}{x}, \quad G_{1, n}^{\prime}\left(K_{1}\right)=0, \\
G_{1, n}\left(K_{1}\right)<0, \quad G_{1, n}\left(K_{1}^{1.106}\right)>0,
\end{gathered}
$$

and then $B_{1}<K_{1}^{1.106}$. So $K_{1}^{1.106}$ is an upper bound for $B_{1}$. 
Now we use a method considered by Maurice Mignotte to solve the problem (see [6]). We know that, for $|x|<1$,

$$
\log (1+x)=x-\frac{\theta}{2} x^{2}, \quad \frac{1}{1+x}=1-x+\theta_{0} x^{2}, \quad 0<\theta, \theta_{0}<1,
$$

and we use the inequalities on $\alpha_{i}$ to obtain respectively

$$
\begin{gathered}
\alpha=n^{2}\left(1+\frac{1}{n}+\frac{1}{n^{2}}+\frac{\theta_{1}}{n^{3}}\right), \beta=n\left(1+\frac{1}{n}+\frac{1}{n^{2}}-\frac{\theta_{2}}{n^{3}}\right), \quad 1<\theta_{1}, \theta_{2}<2, \\
\lambda_{1}=\frac{\alpha+\beta}{\alpha+1 / \beta}=\frac{1+\frac{2}{n}+\frac{2}{n^{2}}+\frac{\theta_{3}}{n^{3}}}{1+\frac{1}{n}+\frac{1}{n^{2}}+\frac{\theta_{4}}{n^{3}}}, \quad 2<\theta_{3}, \theta_{4}<3 .
\end{gathered}
$$

Then we deduce that

$$
\begin{aligned}
& \delta=\frac{\log (\beta)}{\log (\alpha)}=\frac{1}{2}+\frac{1}{4 n \log (n)}+\frac{\vartheta}{8 n^{2} \log (n)}, \quad \text { with } \quad|\vartheta|<3, \\
& \delta_{1}=\frac{\log \left(\lambda_{1}\right)}{\log (\alpha)}=\frac{1}{2 n \log (n)}+\frac{\vartheta_{1}}{4 n^{2} \log (n)}, \quad \text { with } \quad \mid \vartheta_{1}<3
\end{aligned}
$$

Moreover, we have

$$
\left|A_{1}+B_{1} \frac{\log (\beta)}{\log (\alpha)}+\frac{\log \left(\lambda_{1}\right)}{\log (\alpha)}\right|=\left|A_{1}+B_{1} \delta+\delta_{1}\right|<\frac{\tau_{1}}{\log (\alpha)}<\frac{1}{n^{2} \log (n)}, \quad \text { for } n \geq 2 .
$$

Then we obtain

$$
\left\|B_{1}\left(\frac{1}{2}+\frac{1}{4 n \log (n)}\right)+\frac{1}{2 n \log (n)}\right\|<\frac{B_{1} \vartheta+2 \vartheta_{1}}{8 n^{2} \log (n)}<\frac{3 B_{1}+6}{8 n^{2} \log (n)},
$$

where $\|x\|=|x-[x]|=\operatorname{dist}(x, \mathbb{Z})$. As $B_{1}=-2 u_{2}$ is an even integer, we obtain

$$
\left\|B_{1}\left(\frac{1}{2}+\frac{1}{4 n \log (n)}\right)+\frac{1}{2 n \log (n)}\right\|=\left\|\frac{B_{1}+2}{4 n \log (n)}\right\| .
$$

Moreover, we have

$$
\frac{B_{1}+2}{4 n \log (n)}<\frac{63}{256}<\frac{1}{4} \Longleftrightarrow B_{1}<\frac{63}{64} n \log (n)-2 .
$$

We know that $B_{1}$ has an upper bound $K_{1}^{1.106}$, and for $n \geq N_{1}=1.999 \times 10^{18}$, we have

$$
B_{1}<K_{1}^{1.106}<\frac{63}{64} n \log (n)-2
$$

In this case, we obtain

$$
\left\|B_{1}\left(\frac{1}{2}+\frac{1}{4 n \log (n)}\right)+\frac{1}{2 n \log (n)}\right\|=\left\|\frac{B_{1}+2}{4 n \log (n)}\right\|=\frac{B_{1}+2}{4 n \log (n)} .
$$

But we have

$$
\frac{B_{1}+2}{4 n \log (n)}>\frac{3 B_{1}+6}{8 n^{2} \log (n)},
$$

for $n \geq 2$. So this contradicts (4.2), i.e., there does not exist a non-trivial solution of type 1 , for $n \geq N_{1}$. 
4.2. Solutions of type 2. Suppose now $(x, y) \in \mathbb{Z}^{2}$ is a non-trivial solution of type 2 .

Lemma 4.6. We have

$$
\left\{\begin{array}{l}
y\left(-\beta-\alpha-\frac{1}{2 n^{4}}\right)<\gamma_{1}<y(-\beta-\alpha) \\
-y \frac{1}{2 n^{4}}<\gamma_{2}<0 \\
y\left(-\beta-\frac{1}{\alpha}-\frac{1}{2 n^{4}}\right)<\gamma_{3}<y\left(-\beta-\frac{1}{\alpha}\right) \\
y\left(-\beta+\frac{1}{\beta}-\frac{1}{2 n^{4}}\right)<\gamma_{4}<y\left(-\beta+\frac{1}{\beta}\right) .
\end{array}\right.
$$

Proof. The same as the proof of Lemma 4.1.

We use the Siegel identity

$$
0<\frac{\gamma_{3}\left(\alpha_{2}-\alpha_{1}\right)}{\gamma_{1}\left(\alpha_{2}-\alpha_{3}\right)}=1-\frac{\gamma_{2}\left(\alpha_{3}-\alpha_{1}\right)}{\gamma_{1}\left(\alpha_{3}-\alpha_{2}\right)}
$$

to obtain the following linear form in logarithms:

$$
\Lambda_{2}=A_{2} \log (\alpha)+B_{2} \log (\beta)+\log \left(\lambda_{2}\right)=\log \left(1+\tau_{2}\right),
$$

where $A_{2}=-2 u_{2}, B_{2}=-2 u_{3}, \lambda_{2}=\frac{\alpha+\beta}{\beta+\frac{1}{\alpha}}, \tau_{2}=\frac{\gamma_{2}}{\gamma_{1}}\left(\frac{\alpha-1 / \alpha}{1 / \alpha+\beta}\right)$.

We obtain the equivalent of Lemmas 4.2, 4.3, 4.4 and 4.5.

Lemma 4.7. $\Lambda_{2} \neq 0$.

Lemma 4.8. We have

$$
u_{2}<0<u_{3}, \quad 0<\left|u_{2}\right|<u_{3}, \quad \text { and } \quad 0<A_{2}<\left|B_{2}\right| .
$$

Lemma 4.9. We have

$$
\log \left(\Lambda_{2}\right)>-2^{33} \times 3^{7} \times \log (24) \log ^{3}(\alpha \beta) \log \left(\left|B_{2}\right|\right) .
$$

Lemma 4.10. For $n \geq 4$, we have

$$
\log \left(\Lambda_{2}\right)<-1.12\left|B_{2}\right| \log (\alpha) .
$$

So $K_{2}^{1.104}$ is an upper bound for $\left|B_{2}\right|$, and we can use the approach of type 1 to reach a contradiction for $n \geq N_{2}=2.322 \times 10^{18}$. Hence there is no non-trivial solution for $n \geq N_{2}$.

4.3. Solutions of type 3. Suppose that $(x, y) \in \mathbb{Z}^{2}$ is a non-trivial solution of type 3 .

Lemma 4.11. We have

$$
\left\{\begin{array}{l}
y\left(\frac{1}{\alpha}-\alpha-\frac{1}{2 n^{2}}\right)<\gamma_{1}<y\left(\frac{1}{\alpha}-\alpha\right), \\
y\left(\frac{1}{\alpha}+\beta-\frac{1}{2 n^{2}}\right)<\gamma_{2}<y\left(\frac{1}{\alpha}+\beta\right), \\
-y \frac{1}{2 n^{2}}<\gamma_{3}<0, \\
y\left(\frac{1}{\alpha}+\frac{1}{\beta}-\frac{1}{2 n^{2}}\right)<\gamma_{4}<y\left(\frac{1}{\alpha}+\frac{1}{\beta}\right) .
\end{array}\right.
$$

Proof. The same as the proof of Lemma 4.1. 
We use the Siegel identity

$$
0<\frac{\gamma_{2}\left(\alpha_{3}-\alpha_{4}\right)}{\gamma_{4}\left(\alpha_{3}-\alpha_{2}\right)}=1-\frac{\gamma_{3}\left(\alpha_{2}-\alpha_{4}\right)}{\gamma_{4}\left(\alpha_{2}-\alpha_{3}\right)},
$$

to obtain the following linear form in logarithms:

$$
\Lambda_{3}=A_{3} \log (\alpha)+B_{3} \log (\beta)+\log \left(\lambda_{3}\right)=\log \left(1+\tau_{3}\right),
$$

where $A_{3}=-2 u_{3}, B_{3}=2 u_{2}, \lambda_{3}=\frac{1 / \alpha+1 / \beta}{1 / \alpha+\beta}, \tau_{3}=-\frac{\gamma_{3}}{\gamma_{4}}\left(\frac{\beta-1 / \beta}{1 / \alpha+\beta}\right)$.

As before, we obtain the following results.

Lemma 4.12. $\Lambda_{3} \neq 0$.

Lemma 4.13. We have

$$
0<u_{3}<u_{2} \quad \text { and } \quad 0<\left|A_{3}\right|<B_{3} .
$$

Lemma 4.14. We have

$$
\log \left(\Lambda_{3}\right)>-2^{33} \times 3^{7} \times \log (24) \log ^{3}(\alpha \beta) \log \left(B_{3}\right)
$$

Lemma 4.15. For $n \geq 4$, we have

$$
\log \left(\Lambda_{3}\right)<-0.25 B_{3} \log (\alpha)
$$

So $K_{3}^{1.102}$ is an upper bound for $B_{3}$, and we can use the approach of type 1 to reach a contradiction for $n \geq N_{3}=1.168 \times 10^{19}$. Hence there is no non-trivial solution for $n \geq N_{3}$.

4.4. Solutions of type 4. Suppose that $(x, y) \in \mathbb{Z}^{2}$ is a non-trivial solution of type 4 .

Lemma 4.16. We have

$$
\left\{\begin{array}{l}
y\left(-\frac{1}{\beta}-\alpha\right)<\gamma_{1}<y\left(-\frac{1}{\beta}-\alpha+\frac{1}{2 n^{2}}\right) \\
y\left(-\frac{1}{\beta}+\beta\right)<\gamma_{2}<y\left(-\frac{1}{\beta}+\beta+\frac{1}{2 n^{2}}\right) \\
-y\left(-\frac{1}{\beta}-\frac{1}{\alpha}\right)<\gamma_{3}<y\left(-\frac{1}{\beta}-\frac{1}{\alpha}+\frac{1}{2 n^{2}}\right) \\
0<\gamma_{4}<y \frac{1}{2 n^{2}}
\end{array}\right.
$$

Proof. The same as the proof of Lemma 4.1 .

We use the Siegel identity

$$
0<\frac{\gamma_{1}\left(\alpha_{4}-\alpha_{3}\right)}{\gamma_{3}\left(\alpha_{4}-\alpha_{1}\right)}=1-\frac{\gamma_{4}\left(\alpha_{1}-\alpha_{3}\right)}{\gamma_{3}\left(\alpha_{1}-\alpha_{4}\right)},
$$

to obtain the following linear form in logarithms:

$$
\Lambda_{4}=A_{4} \log (\alpha)+B_{4} \log (\beta)+\log \left(\lambda_{4}\right)=\log \left(1+\tau_{4}\right) .
$$

where $A_{4}=2 u_{2}, \quad B_{4}=2 u_{3}, \quad \lambda_{4}=\frac{1 / \alpha+1 / \beta}{\alpha+1 / \beta}, \quad \tau_{4}=-\frac{\gamma_{4}}{\gamma_{3}}\left(\frac{\alpha-1 / \alpha}{\alpha+1 / \beta}\right)$.

We obtain the following results.

Lemma 4.17. $\Lambda_{4} \neq 0$.

Lemma 4.18. We have

$$
u_{3}<0<u_{2}, \quad 0<u_{2}<\left|u_{3}\right|, \quad 0<A_{4}<\left|B_{4}\right| \text {. }
$$


Lemma 4.19. We have

$$
\log \left(\Lambda_{4}\right)>-2^{33} \times 3^{7} \times \log (24) \log ^{3}(\alpha \beta) \log \left(\left|B_{4}\right|\right) .
$$

Lemma 4.20. For $n \geq 4$, we have

$$
\log \left(\Lambda_{4}\right)<-0.27\left|B_{4}\right| \log (\alpha) .
$$

So $K_{4}^{1.104}$ is an upper bound for $B_{4}$, we can use the approach of type 1 to reach a contradiction for $n \geq N_{4}=1.169 \times 10^{19}$. Hence there is no non-trivial solution for $n \geq N_{4}$.

Now let us put $N=\max \left\{N_{1}, N_{2}, N_{3}, N_{4}\right\}$.

Theorem 4.21. For $n \geq N=1.191 \times 10^{19}$, with $n, n+2, n^{2}+4$ square-free, the equation

$$
\Phi_{n}(x, y)=x^{4}-n^{2} x^{3} y-\left(n^{3}+2 n^{2}+4 n+2\right) x^{2} y^{2}-n^{2} x y^{3}+y^{4}=1,
$$

has only the trivial solutions $(0,1),(0,-1),(1,0),(-1,0)$.

\section{Part II: Small values of $n$}

For small values of $n$, the previous method is not successful, so we use a method of Y. Bilu and G. Hanrot (see [2]). It is a computational method which allows us to determine a Baker's bound for the exponents $b_{i}=u_{i}$ (see (3.2)), to reduce this bound, and then to solve completely the equation. We obtain the following result.

Theorem 5.1. For $n \leq 5 \times 10^{6}$, with $n, n+2, n^{2}+4$ square-free, the equation

$$
\Phi_{n}(x, y)=x^{4}-n^{2} x^{3} y-\left(n^{3}+2 n^{2}+4 n+2\right) x^{2} y^{2}-n^{2} x y^{3}+y^{4}=1
$$

has only the trivial solutions $(0,1),(0,-1),(1,0),(-1,0)$.

Here are a few remarks about the computation. In order to reduce Baker's bound, we determined the expressions $X_{i}, c_{i}, \delta_{i}$, which allows to determine a Baker's bound for the exponents $b_{i}$, to make the reduction, see [2], and we wrote a program in PARI/GP. We know that $\varepsilon<n+1, \alpha<(n+1)^{2}$ and $\beta<n+2$, so we put $n_{2}=\left(n_{1}+2\right)\left(\left|b_{1}\right|+2\left|b_{2}\right|+\left|b_{3}\right|+1\right)+50$ as the computation's precision, where $n_{1}$ is the number of digits of $n+2$.

After almost 3 reductions, we obtain $B_{0} \leq 4$ and $X_{3}=1$. The integral solutions $(x, y)$ verifying $|x| \leq X_{3},|y| \leq X_{3}$ are: $(-1,0),(0,-1),(0,1),(1,0)$. In fact, we let $b_{1}, b_{2}, b_{3}$ run between $-B_{0}$ and $B_{0}$, and we observed the exponents associated with the integral solutions. Then we obtain:

for types 1 and 2: $b_{1}=b_{2}=b_{3}=0, x=1, y=0$, so $(1,0)$ is a solution;

for types 3 and $4: b_{1}=0, b_{2}=1, b_{3}=0, x=0, y=-1$, so $(0,-1)$ is a solution.

The property (b) of section 2 gave us the other solutions. The computations were done with a SUN PARC ULTRA1, and for each value of $n$, the time of computation is between 3 and 5 seconds.

\section{Acknowledgments}

I thank Professors Cornelius Greither, Günther Frei, Maurice Mignotte of my dissertation committee for their helpful advice, and particularly Professor Claude Levesque for, amongst many things, supervising me. I also thank the referee for the suggestions on an earlier draft of the manuscript. 


\section{REFERENCES}

1. A. Baker, G. WÜstholz, Logarithmic forms and group varities, J. reine angew. Math. 442 (1993), 19-62. MR 94i:11050

2. Y. Bilu, G. Hanrot, Solving Thue equations of high degree, J. Number Theory 60 (1996), 373-392. MR 97k:11040

3. G. HANRot, Résolution effective d'équations diophantiennes: algorithmes et applications, Thèse, Université Bordeaux 1, 1997.

4. O. Lechcheux, Familles de corps de degré 4 et 8 liées à la courbe modulaire $X_{1}(16)$, Séminaire de Théorie des Nombres, Paris, 1991-92, 89-105. MR 95i:11060

5. G. Lettr, A. Pethő, Complete solution of a family of quartic Thue equations, Abh. Math. Sem. Univ. Hamburg 65 (1995), 365-383. MR 96h:11019

6. M. Mignotte, Verification of a conjecture of E. Thomas, J. Number Theory 44 (1993), 172-177. MR 94m:11035

7. M. Mignotte, A. Pethö, F. Lemmermeyer, On the family of Thue equations $x^{3}-$ $(n-1) x^{2} y-(n+2) x y^{2}-y^{3}=k$, Acta Arithmetica 76 (1996), 245-269. MR 97k:11039

8. M. Mignotte, A. Рethö, R. Roth, Complete solutions of a family of parametric quartic Thue and index form equations, Math. Comp. 65, No. 213 (1996), 341-354. MR 96d:11034

9. А. Ретнӧ, Complete solutions to families of quartic Thue equations, Math. Comp. 57, No. 196 (1991), 777-798. MR 92e:11023

10. E. Thomas, Complete solutions to a family of cubic diophantine equations, J. Number Theory 34 (1990), 235-250. MR 91b:11027

11. E. Thomas, Solutions to certain families of Thue equations, J. Number Theory 43 (1993), 319-369. MR 94b:11028

12. A. TogBé, Sur la résolution de familles d'équations diophantiennes, Thèse de doctorat, Université Laval, Québec, Canada, Décembre 1997.

13. L. C. Washington, A family of cyclic quartic fields arising from modular curves, Math. Comp. 57, No. 196 (1991), 763-775. MR 92a:11120

Département de Mathematiques et de Statistique, Université Laval, Québec, Québec, G1K 7P4 CANADA

Current address: Department of Mathematics \& Computer Science, Greenville College, 315 E.

College Avenue, Greenville, IL 62246

E-mail address: atogbe@greenville.edu 